Running head: rTMS AND ATTENTIONAL BIAS

\title{
The Effects of High Frequency rTMS on Negative Attentional Bias are Influenced by Baseline State Anxiety.
}

Vanderhasselt, M.A., Baeken, C., Hendrickx, M., \& De Raedt, R. (in press). The Effects of High Frequency rTMS on Negative Attentional Bias are Influenced by Baseline State Anxiety. Neuropsychologia

Marie-Anne Vanderhasselt ${ }^{* 1}$, Chris Baeken ${ }^{2}$, Marianne Hendricks $^{1}$, \& Rudi De Raedt ${ }^{1}$

${ }^{1}$ Department of Experimental Clinical and Health Psychology, Ghent University, Belgium

${ }^{2}$ Department of Psychiatry, University Hospital UZ-Brussel, Belgium

* Corresponding Author: Marie-Anne Vanderhasselt, Ph. D. Department of Experimental and Clinical Health Psychology, Ghent University, Henri Dunantlaan 2, 9000 Gent, Belgium. Tel: 001 (9) 26464 44; Fax: 001 (9) 26464 87. E-mail: MarieAnne.Vanderhasselt@Ugent.be

Word count: 6941 


\begin{abstract}
High Frequency (HF) repetitive Transcranial Magnetic Stimulation (rTMS) of the right Dorsolateral Prefrontal Cortex (DLPFC) has been shown to induce an attentional bias towards threatening information in healthy adults, associated with decreased activation in the right DLPFC and increased activation in the right amygdala. Additionally, it has been shown that healthy individuals with higher state anxiety portray similar negative attentional biases and cortico-subcortical activation patterns to those induced by HF-rTMS of the right DLPFC. Therefore, the aim of this study is to investigate whether inter-individual differences in state anxiety levels prior to the administration of HF-rTMS of the right DLPFC might be related to the degree to which rTMS induces such a negative attentional bias in healthy volunteers. We administered HF-rTMS of the right DLPFC to a group of 28 healthy female individuals. In line with previous research, a single session of HF-rTMS of the right DLPFC induced an attentional bias towards threatening information. Moreover, self-report measures of state anxiety (STAIState) prior to stimulation correlated positively with the magnitude of the induced attentional bias. More specifically, we found that healthy individuals who scored higher on self-reports of state anxiety acquired more attentional bias towards negative information after HF-rTMS. Therefore, the effects of a single placebo-controlled rTMS session of the right DLPFC is consistent with the effects of a disrupted prefrontal-amygdala circuitry. The effects on attentional bias are largest in those participants reporting higher state anxiety scores, possibly because underlying amygdala activation is highest.
\end{abstract}

Key words: Attentional Bias, rTMS, State Anxiety, DLPFC 


\section{The Effects of High Frequency rTMS on Negative Attentional Bias are Moderated by Baseline State Anxiety}

\section{Introduction}

Although repetitive Transcranial Magnetic Stimulation (rTMS) is considered a relatively new technology (Pascual-Leone et al., 1998), the number of studies using this technique has increased enormously in recent decades. As a non-invasive tool for the stimulation of the human cerebral cortex, rTMS over the dorsolateral prefrontal cortex (DLPFC) holds promise not only

for therapeutic advances, but also for research insights into cognitive information processes (e.g. Vanderhasselt, De Raedt, Baeken, Leyman, \& D’Haenen, 2006). Very recently, placebocontrolled studies in healthy volunteers demonstrated that a single session of High Frequency (HF)-rTMS of the right DLPFC caused an increased attentional bias for threatening information (angry faces) (Leyman, De Raedt, Vanderhasselt, \& Baeken, 2009). In a follow-up study combining rTMS and fMRI, we observed that this induced negative attentional bias was independent of any changes in self-reported emotions but was associated with decreased activation within the right DLPFC, dorsal anterior cingulate cortex and left superior parietal gyrus, combined with increased activity within the right amygdala (De Raedt et al., 2010).

Interestingly, this experimentally induced attentional bias and the related corticosubcortical changes are very similar to the cognitive and neural features that characterize individuals with high self-reported anxiety states. At the cognitive level, anxiety is related to an increased attentional bias, specifically an impaired disengagement from angry faces (e.g. Fox, Russo, \& Dutton, 2002; Fox, Russo, \& Georgiou, 2005; Fox, Russo, Bowles, \& Dutton, 2001; Georgiou et al., 2005). These difficulties in disengaging attention can be seen as a failure to recruit attentional control processes, associated with decreased prefrontal activation, in order to 
down-regulate increased amygdala activation (for a review, see De Raedt \& Koster, 2010; Nitschke \& Heller, 2005). Indeed, at the neural level, Bishop, Duncan, Brett, \& Lawrence (2004a) reported that healthy people with higher state anxiety levels showed reduced recruitment of the lateral PFC (ventrolateral and dorsolateral) when processing task-irrelevant threatening distractors. Moreover, Fales and coworkers (Fales, Barch, Rundle, Mintun, \& Snyder, 2008) observed that healthy, non-depressed participants showed increased activity in right DLPFC (Brodmann areas 46/9) when asked to ignore fear-related stimuli in an attention task. These data appear to imply that a negative attentional bias is caused by reduced DLPFC activation and related top-down attentional control, resulting in increased amygdala activation during the presentation of threatening information. This involvement of the DLPFC in down-regulating the amygdala is also observed in studies where participants are asked to cognitively regulate (e.g., reappraise) their emotional responses (Ochsner, Bunge, Gross, \& Gabrieli, 2002).

Taken together, these cognitive and neural characteristics that are associated with increased state anxiety in healthy individuals are very similar to the cognitive and neural effects induced by rTMS of the right DLPFC in healthy controls (De Raedt et al., 2010). An interesting research question remains as to whether inter-individual differences in state anxiety may increase the degree to which rTMS has an effect on attentional bias for threatening information in a group of healthy individuals. This is because a single session of rTMS interrupts the DLPFC-amygdala circuitry during disengagement from threatening information, leading to higher amygdala activation (De Raedt et al., 2010). This interruption may have a different effect for high and low anxiety participants because it is known that higher state anxiety is related to augmented amygdala activation (Davidson, 2002). This increased amygdala activation is normally (e.g., in healthy volunteers) regulated by enhanced top-down DLPFC activation, leading to an absent negative attentional bias (Bishop et al., 2004a). However, because a disruption of the DLPFC- 
amygdala circuitry by a single rTMS session leads to increased amygdala activation associated with an increased negative attentional bias, this inducted bias may be more pronounced in higher anxious individuals, because their underlying amygdala activation is highest. Therefore, the aim of this study is to investigate whether levels of state anxiety prior to the administration of rTMS have an influence on the induction of a negative attentional bias following this brain stimulation procedure in healthy volunteers.

Hence, in this study we administered HF-rTMS of the right DLPFC and correlated selfreport measures of state anxiety to the degree of the induced attentional bias. In line with De Raedt et al. (2010), we used an emotional modification of the exogenous cueing task (ECT: see Koster, De Raedt, Goeleven, Franck, \& Crombez, 2005; Posner, 1980) to measure attentional bias. In the ECT task, a target appears at one of two spatial locations, preceded by a cue at the same ('valid trial') or the opposite location ('invalid trial'). Exogenous cues that are presented for a short duration $(<300 \mathrm{~ms})$ facilitate a response to target stimuli on valid trials, whereas a reaction time cost is observed on invalid trials. The emotional value of the cue is angry or neutral, which allows a comparison to be made of the reaction time benefits in responding to valid versus invalid emotionally negative (angry) cues compared to emotionally neutral cues. The cue validity (CV: e.g. Fox et al., 2002) refers to this difference between valid and invalid trials; a higher score indicates that attention is drawn to or held at the spatial location of the cue and stands for an increased attentional bias. It is known that anxious individuals show an enhanced cue validity effect, whereas no such pattern is observed in non-anxious individuals (for a review, see Cisler \& Koster, 2010).

Based on prior literature and the studies carried out by our lab, we predicted that a single session of HF-rTMS of the right DLPFC would have no influence on mood measurements 
(Baeken, Leyman, De Raedt, Vanderhasselt, \& D’Haenen, 2006, 2008; Mossiman, Rihs, Engeler, Fish, \& Schlaepfer, 2000). On the other hand, in line with De Raedt et al. (2010), we predicted that a single session of HF-rTMS of the right DLPFC would induce increased attention for emotionally threatening information (i.e. a negative attentional bias). Moreover, we predicted that the effects of rTMS on an induced negative attentional bias would be related to the level of state anxiety prior to stimulation. More specifically, we hypothesized that attentional bias for negative information following rTMS would be elevated in individuals with higher levels of state anxiety prior to stimulation. This is because rTMS will weaken the DLPFC top-down control of the amygdala, leading to increased amygdala activation. This disruption will be associated with negative attentional biases, specifically in those participants scoring high on baseline state anxiety.

\section{Methods}

\subsection{Participants}

Postings on the university website were used to recruit a group of 28 female participants with a mean age of $22.29(S D=2.58$; age range: $18-29)$. We selected only female participants because women show stronger brain activation in emotional tasks, particularly in areas related to subjective feelings (Wager, Phan, Liberzon, Taylor, 2003). They tend to rate their emotions more intensely and demonstrate greater facility in decoding non-verbal messages than males (Killgore \& Yurgelun-Todd, 2001). Moreover, all previous rTMS volunteer studies carried out by our lab included only female volunteers.

Based on the safety guidelines (Rossi et al., 2009), all participants underwent a standard physical and mental examination performed by a trained psychiatrist. No current and/or history of psychiatric disorders was confirmed using the International Neuropsychiatric Interview 
(M.I.N.I; Sheehan et al., 1997). Moreover, the absence of current depressive symptoms was confirmed using the Beck Depression Inventory (BDI-II; Beck, Steer, \& Brown, 1996; cutoff 14) $(M=3.14: S D=3.00)$, and right handedness was confirmed through a well validated Dutch scale of Van Strien (2001). This experiment was part of a larger project investigating other neurocognitive markers. The study protocol was approved by the local Medical Ethics Committee of the University Hospital (UZBrussel) of the Vrije Universiteit Brussel (V.U.B.). All participants received financial compensation.

\subsection{Procedure}

A SHAM (placebo-)controlled 'double' blind crossover design was used. Every morning before the start of the experiment (for SHAM and real rTMS), all volunteers were assessed using the state version of the State-Trait Anxiety Inventory (STAI; Spielberger, 1983; translated into Dutch by Van der Ploeg, 1982). Subsequently, participants performed an emotional modification of the Exogenous Cueing Task (ECT: Posner, 1980), followed by either real or SHAM stimulation of the right DLPFC. Finally, participants performed the ECT for the second time. The order of the stimulation sessions (real versus SHAM) was counterbalanced. Fourteen participants first received real HF-rTMS before SHAM and the fourteen other volunteers received SHAM followed by the real condition. To avoid carry-over effects from the previous stimulation, the second session was carried out after an interval of one week. All volunteers were stimulated within the same time schedule, between 10 am and $2 \mathrm{pm}$. All participants wore earplugs and were blindfolded to guarantee blindness to the stimulation condition.

Subjective mood ratings were recorded using the Profile of Mood States (POMS; Wald \& Mellenbergh, 1990$)$ at three time points: baseline $\left(\mathrm{T}_{0}\right)$, immediately after stimulation $\left(\mathrm{T}_{1}\right)$, and after task performance (approximately 30 minutes after stimulation, $\mathrm{T}_{2}$ ). 


\subsection{Materials and Methods}

\subsubsection{Mood ratings}

The Profile of Mood States (POMS; Wald \& Mellenbergh, 1990) was administered to measure mood states. The POMS consists of 32 items over five mood dimensions: (1) depressive feelings (8 items: minimum score 0 ; maximum score 32$)$; (2) fatigue (6 items: minimum score 0 ; maximum score 24); (3) tension (6 items: minimum score 0; maximum score 24); (4) anger (7 items: minimum score 0 ; maximum score 28); and (5) vigor (5 items: minimum score 0; maximum score 20). For each item, participants were asked to indicate how they felt at that moment out of a five-point Likert scale $(0=$ absolutely not; $4=$ a lot $)$. The POMS is reliable and well validated (Wald \& Mellenbergh, 1990).

\subsubsection{Anxiety measure}

To measure state anxiety, the state version of the STAI was administered (STAI; Spielberger, 1983; translated into Dutch by Van der Ploeg, 1982). This questionnaire consists of twenty statements to be rated on a 4-point scale. The reliability and validity of the STAI is well documented.

\subsubsection{Exogenous cueing task}

Attentional processing of threatening information was measured using an emotional modification of the exogenous cueing task (ECT: Posner, 1980). The instructions were explained verbally and presented on the computer screen. Participants were instructed to respond as quickly

and as accurately as possible to the location of a small, black square $(1.1$ by $1.1 \mathrm{~cm}$; visual angle: $\left.1.1^{\circ}\right)$ which would appear on the left or right side of a fixation cross. They were informed that a cue would precede the presentation of the target and that the cue was not predictive for the target 
location. It was emphasized that attention should be directed towards a fixation cross during the experiment. Responses could be made by pressing one of two assigned keys of a response box connected to a personal computer (press left key with left finger and right key with right finger). Inquisit software (Millisecond Software, 2001, Version 1.33) was used to record response accuracy and latencies. They were seated $+/-55 \mathrm{~cm}$ from the 15.4 inch computer screen.

Before the target appeared, a picture of an angry or neutral face was presented, equally often on the left and right side of the screen. The location of the picture cued the spatial location of the target in $2 / 3$ of the trials (valid cue) and incorrectly cued the location of the target in the remaining 1/3 of the trials (invalid cue). Each trial started with the presentation of 2 white frames ( $7 \mathrm{~cm}$ high by $7 \mathrm{~cm}$ wide; visual angle: $7.3^{\circ}$ ) located on both sides of the screen, on a black background. They remained on the screen throughout the entire trial. The middle of each of these rectangles was at a distance of $7 \mathrm{~cm}\left(7.3^{\circ}\right.$ visual angle $)$ from the fixation cross. The presentation duration of these frames was jittered over a range of intervals from $100 \mathrm{~ms}$ to $2000 \mathrm{~ms}$. Next, a fixation cross appeared in the middle of the screen as the remainder of the trial presentation. Five hundred ms after presentation of the fixation cross, a picture of a neutral or an angry face was presented for $200 \mathrm{~ms}$ in place of one of the 2 white frames. Next, after a mask of $50 \mathrm{~ms}$, the target appeared for $1500 \mathrm{~ms}$ or until the subject responded (Figure 1). Subjects first completed 20 practice trials, followed by 189 test trials. The order of trials was fixed.

The stimuli were taken from The Karolinska Directed Emotional Faces (KDEF) database (Lundqvist, Flykt, \& Öhman, 1998). Before selection, all pictures were adjusted to exclude interference of background stimuli (hair, clothing) so that only the face was presented. In addition, all pictures were in color and adjusted to the same size (326 X 326 pixels). A total of 20 neutral and 20 angry faces were selected from a validation study of the KDEF picture set 
(Goeleven, De Raedt, Leyman, \& Verschuere, 2008). Pictures of angry faces were selected if more than $75 \%$ of the participants categorized them as angry with an average intensity rating higher than 6 (intense). Pictures of neutral faces were selected if more than $75 \%$ of the raters categorized them as neutral and if they had an average intensity rating that was moderate $(>4)$. Five neutral and 5 angry faces were presented in the practice phase; the remaining pictures of 15 neutral and 15 angry faces were presented in the test trials.

This cognitive task enabled us to investigate attentional biases for emotional information by calculating a Cue Validity index $(\mathrm{CV})$. The $\mathrm{CV}$ was calculated using the following formulae (Fox et al., 2002, Leyman, De Raedt, Schacht, \& Koster, 2007): (1) Cue-Validity-index (CVi) = RT invalid cue $-\mathrm{RT}$ valid cue; (2) CV = CVi for angry $-\mathrm{CVi}$ for neutral. As a result, a positive score on this latter score indicates increased attentional bias for angry faces in comparison to neutral control faces (e.g. a higher CV indicates an increased attentional bias for negative information). It is important to note that, using the ECT, more specific components of attention attentional engagement and disengagement - can be examined. However, some possible confounds have been noted in the analyses of these components (Mogg, Holmes, Garner, \& Bradley, 2008). Therefore, we chose to limit our analyses to the cue validity component as our main index of attentional bias. ${ }^{1}$.

[Insert Figure A.1. About Here]

\subsubsection{Stimulation protocol}

\footnotetext{
${ }^{1}$ The results in the present study were very similar when using the disengagement scores as compared to the cue validity index.
} 
We used a Magstim high-speed magnetic stimulator (Magstim®, Corpumed, Sheffield, UK) equipped with a figure-of-eight-shaped double 70mm coil. The rTMS stimulation parameters were well within the established safety guidelines (Rossi et al., 2009). To obtain individual anatomical information, all participants underwent a T1-weighted MRI (3D-TFE, voxel size $1 \mathrm{x} 1 \mathrm{x} 1 \mathrm{~mm}$ ) of the brain using a $1.5 \mathrm{~T}$ Intera MR scanner (Philips, Best, the Netherlands). All post-processing was carried out on a ViewForum console (Philips, Best, the Netherlands). In order to accurately target the right DLPFC, the precise stimulation site and position of the coil were determined using MRI non-stereotactic guidance (Philips Intera, Best, the Netherlands). The DLPFC was located visually on the 3D surface rendering of the brain based on the subject's own gyral morphology, marking the middle part of the midfrontal gyrus as the centre of the left or right DLPFC, area 9/46 (MNI coordinates: $-45,30,31$ ). Based on the coordinates of this point, the precise stimulation site on the skull (right DLPFC) was marked and stimulated. The corresponding coil position was found by determining the perpendicular projection of this point on the scalp (Peleman et al., 2010).

Stimulation intensity of $110 \%$ of the motor threshold (MT) at rest of the right abductor pollicis brevis (APB) muscle was established using EMG. HF-rTMS $(10 \mathrm{~Hz})$ was delivered over the right DLPFC using the following parameters: 40 trains of 3.9 seconds' duration, separated by an intertrain interval of 26.1 seconds, resulting in 1560 pulses per session. The total stimulation time was approximately 20 minutes.

Each subject received one SHAM placebo stimulation and one real rTMS session. The order of the first stimulation session, i.e. real rTMS stimulation vs. SHAM stimulation, was assigned by a crossover design. SHAM stimulation was performed at the identical place on the skull, but the figure-of-eight-shaped coil was held at an angle of $90^{\circ}$, resting on the scalp with 
only one wing, in compliance with recent SHAM guidelines (Loo et al., 2000). During stimulation, all participants wore earplugs and were blindfolded to guarantee 'optimal' blinding.

\section{Results}

The entire statistical analysis was performed using the SPSS software package (version 16.0). Where necessary, we applied the Greenhouse-Geisser correction to ensure the assumption of sphericity. Effect sizes are reported in the form of partial eta-squared ( $\eta 2)$. In line with Cohen's (1988) guidelines, a $\eta_{\mathrm{p}}^{2}$ of .01,.10, and .25 was used as a threshold to define small, medium, and large effects, respectively.

\subsection{Anxiety scores}

STAI state baseline scores were not significantly different between real rTMS and SHAM, $t(27)=1.60, p=0.12$. For statistical analysis, we calculated the mean STAI state scores over two stimulation days $(M=29.54 ; S D=4.57$; range $=21.5-40)$. Because of missing STAI scores on one of the two stimulation days (rTMS or SHAM) in two subjects, we replaced the missing value by the value of the other STAI score.

\section{2. $\quad$ Effects on mood}

Table 1 shows the mean POMS scores (with Standard Deviation $(S D)$ ) on all five subscales immediately before stimulation $\left(\mathrm{T}_{0}\right)$, immediately after stimulation $\left(\mathrm{T}_{1}\right)$, and, finally, half an hour after stimulation $\left(\mathrm{T}_{2}\right)$. To investigate changes in mood as an effect of stimulation, the data of each subscale were compressed to a multivariate ANOVA (MANOVA) with Stimulation (rTMS, SHAM) and Time $\left(\mathrm{T}_{0}, \mathrm{~T}_{1}, \mathrm{~T}_{2}\right)$ as two within-subjects factors. We obtained a main effect of Time, $F(10,18)=3.66, p<.01, \eta^{2} p=.67$. The other main effect of Stimulation 
and the interaction effect yielded no significant effect, $F_{\mathrm{s}}<1.72, p \mathrm{~s}>.17$. Univariate ANOVAs revealed that the main effect of Time was significant for the subscales Tension, $F(2,54)=4.40$, $p<.01, \eta_{p}^{2}=.21$, and Vigor, $F(2,54)=3.88, p=.03, \eta_{p}^{2}=.13$. This means that, although there was no difference between real and placebo stimulation, participants felt tenser and more vigorous after receiving stimulation.

[Insert Table B.1. About Here]

\subsubsection{Effect of STAI scores on mood changes}

To investigate the effects of anxiety, the data of each subscale were compressed to a MANCOVA with Stimulation (rTMS, SHAM) and Time (pre, post 1, post 2) as within-subjects factors and the STAI score as a covariate. We found no effects involving the STAI, $F \mathrm{~s}<1.74, p \mathrm{~s}$ $>.15$, which indicates that levels of anxiety had no influence on the effects of rTMS on mood.

\section{3. $\quad$ Effects on cue validity}

Table 2 shows the mean reaction times and SD on the ECT. Trials in which participants 1) made an incorrect response and/or 2) did not respond within a window of $2.5 S D$ of individuals' mean per condition were excluded (rTMS session: 5.51 $\pm 2.85 \%$; SHAM session: $4.30 \pm 3.64 \% ; t(27)=.88, p=.28$.

The data were analyzed using a 2 × 2 repeated measures ANOVA with Stimulation (rTMS, SHAM) and Time (pre, post) as the within-subjects factors and using the cue validity score $(\mathrm{CV})$ as the dependent variable. The main effects of Stimulation, $F(1,27)=11.67, p<.01$, $\eta_{\mathrm{p}}^{2}=.30$, and Time, $F(1,27)=7.71, p=.01, \eta_{p}^{2}=.22$, were significant. Moreover, we obtained a significant interaction between Stimulation and Time, $F(1,27)=4.72, p=.04, \eta_{p}^{2}=.15$. Paired 
t-tests demonstrated that participants had increased attention for angry faces (higher CV) postrTMS versus pre-rTMS, $t(27)=4.07, p<.001$, but not post-SHAM versus pre-SHAM, $t(27)=$ $.92, p=.37$. Moreover, CV effects were not significantly different between rTMS and SHAM before stimulation, $t(27)=.89, p=.38$, but yielded a significant effect post stimulation, $t(27)=$ $4.08, p<.001$.

[Insert Table B.2. About Here]

\subsubsection{Effect of STAI scores on cue validity}

To investigate the effects of anxiety, we used an ANCOVA with Stimulation (rTMS, SHAM) and Time (pre, post) as within-subjects factors and the STAI score as a covariate. CV scores were used as the dependent variables. For this analysis, we obtained a significant interaction between Stimulation and Time, $F(1,26)=7.25, p=.01, \eta^{2}=.22$. Most important, the three-way interaction including the STAI scores as a covariate was also significant, $F(1,26)$ $=9.66, p<.01, \eta_{p}^{2}=.27$. No other main or interaction effects were significant, $F_{\mathrm{s}}<1.02, p \mathrm{~s}>$ .32. To further explore this interaction effect, we calculated the difference in CV scores before and after stimulation: $\mathrm{CV}_{\text {post }}$ minus $\mathrm{CV}_{\text {pre. }}$ As a result, the higher this latter score, the more attentional bias for negative information increased after stimulation. STAI scores correlated with the difference score pre-post rTMS, $r(28)=.53 ; p<.01$, but not with the difference score prepost SHAM, $r(28)=-.13 ; p=.53$. These findings indicate that the higher the STAI state scores, the more HF-rTMS increased the attentional bias for angry faces (see Figure 2 for a scatter plot). Interestingly, STAI state scores correlated with the CV scores after rTMS, $r(28)=.46 ; p=.01$, but this correlation was not significant before rTMS or before or after SHAM, $r$ s $<31, p s>11$.

[Insert Figure A.2. About Here] 


\section{Discussion}

The aim of our study was to investigate whether inter-individual differences in state anxiety had an influence on the degree to which HF-rTMS of the right DLPFC induces a negative attentional bias in healthy volunteers.

As predicted, a single session of HF-rTMS of the right DLPFC had no effect on selfreported emotions. This is in line with the literature, which reports no changes in mood after a single session of HF-rTMS (Baeken et al., 2006; 2008; Mossiman et al., 2000). Additionally, in line with previous research (Leyman et al., 2009; De Raedt et al., 2010), a single session of HFrTMS of the right DLPFC induced attentional biases toward threatening information on the group as a whole, as evidenced by increased CV after rTMS but not after SHAM. In other words, participants demonstrated an increased attentional bias for threatening information after HFrTMS and these effects cannot be attributed to changes in mood state induced by rTMS.

The present results go beyond these prior findings, indicating that baseline levels of state anxiety have a significant effect on the induction of a negative attentional bias. More specifically, higher state anxiety scores correlated positively with the induced attentional bias for threatening information following HF-rTMS in healthy volunteers. This correlation was observed only after real stimulation and not after placebo SHAM stimulation. It is important to note that the tension subscale (as well as all the other subscales) of the POMS showed no temporal variation that was different between the day they received rTMS and the day they received SHAM. Tension and vigor increased equally after receiving real stimulation and SHAM. Moreover, covariance analysis showed that the level of state anxiety was not related to the effects of rTMS on mood measures. Without this information, one could argue that the "rTMS procedure" and not the "SHAM procedure" may have induced higher levels of stress for 
anxious individuals, leading to changes in attentional bias (Baeken, Vanderhasselt, De Raedt, 2010).

Interestingly, there was no correlation between STAI state scores and attentional bias before stimulation (SHAM and real HF-rTMS). Although this is not in line with existing literature (Fox et al., 2001; 2002; 2005; Georgiou et al., 2005), other previous studies did not report differences in attentional bias for emotional information based on anxiety scores in healthy students (e.g. Koster, Leyman, De Raedt, \& Crombez, 2006). One possible explanation is that most of our healthy participants reported overall low STAI state scores. Indeed, in the present study the median score of STAI-state was 30.25, which is lower than the scores reported in other studies (e.g. Fox et al., 2005: median $=37.5$ ). In fact, we specifically chose a nonclinical sample because anxious individuals might already demonstrate a higher attention bias toward threat prior to stimulation, which might conceal the effects of rTMS on attentional bias due to a ceiling effect.

These results have important theoretical implications. When we combine the research findings presented by De Raedt et al. (2010) and the present findings, it would appear that there is interaction between baseline state anxiety and the induction of an attentional bias towards threat. It is known that this increased attentional bias following rTMS is associated with a decreased down-regulation of the DLPFC, leading to increased amygdala activation during the presentation of threatening information (De Raedt et al., 2010). The present findings suggest that this reduced down-regulation from the DLPFC on the amygdala is most pronounced in those participants reporting the highest state anxiety prior to stimulation. This higher baseline state anxiety would theoretically be related to increased amygdala activation, resulting in a higher negative attentional bias. However, in healthy, non-depressed individuals amygdala hyper- 
activation initiates a negative feedback signal to the DLPFC (Siegle, Steinhauer, Thase, Stenger, Carter, 2002; Taylor \& Fragopanagos, 2005), resulting in increased DLPFC activation to downregulate the amygdala to prevent the development of attentional biases and negative affect. It appears to be the case that in the present non-depressed sample, prior to stimulation, the interaction between amygdala and DLPFC is in balance, which is associated with an absent negative attentional bias, including in those participants reporting higher on state anxiety. Conversely, rTMS appears to disrupt this circuitry, associated with a negative attentional bias (De Raedt et al., 2010). Most important, the present research findings add to the literature, demonstrating that the effects of this circuitry disruption are largest in those participants scoring high on state anxiety, possibly because of highest underlying amygdala activation. The current findings imply that, in a non-clinical sample, inter-individual differences in state anxiety should be taken into account when investigating the effects of rTMS on the negative attentional bias. This is because the presence or absence of a negative attentional bias depends on the amygdala down-regulation supported by the DLPFC interconnection.

Ultimately, dysfunctional top-down attentional control for emotional information and related decreased activation in the prefrontal cortex appear to be crucial underlying working mechanisms in the etiology of increased anxiety (for a review, see Bar-Haim, 2010; Hakamata et al., 2010). In healthy volunteers, MacLeod, Rutherford, Campbell, Ebsworthy, \& Holker (2002) trained healthy volunteers to acquire an attentional bias towards threat and observed an increased stress response. Interestingly, a recent study observed that this attentional training to induce a negative attentional bias in healthy volunteers correlated with decreased activation in lateral prefrontal brain areas (Browning, Holmes, Murphy, Goodwin, \& Harmer, 2009). In our study, we used HF-rTMS of the right DLPFC to induce an attentional bias towards threat and observed similar effects as the effects that were found in the attentional bias training. Indeed, one session 
of HF-rTMS of the right DLPFC induced a negative attentional bias with a magnitude dependent on baseline state anxiety levels, and both (rTMS and anxiety) have been associated with reduced activation in the right DLPFC (De Raedt et al., 2010 ). These rTMS findings might be especially germane in the light of the existing therapeutic applications of multiple sessions of left rTMS as a treatment for anxiety disorders (for a review, see Pallanti \& Bernardi, 2009; see also, Boggio, et al, 2010; Dresler et al., 2009). Indeed, multiple left-sided rTMS sessions have been associated with a decreased attentional bias for negative faces (Leyman, De Raedt, Vanderhasselt, \& Baeken, 2011).

A limitation of the present study should be highlighted. Although we use the neural dynamics that were observed in a prior study (De Raedt et al., 2010) to make inferences based on our empirical findings, no neural data were collected in the present study. De Raedt et al. (2010) observed that the induced negative attentional bias was associated with decreased activation within the right DLPFC, dorsal anterior cingulate cortex and left superior parietal gyrus, combined with increased activity within the right amygdala (De Raedt et al., 2010). While rTMS of the right DLPFC induced a similar negative attentional bias in the present study compared to the study of De Raedt et al. (2010), it remains unclear whether the level of state anxiety actually influences the effect of rTMS on the inter-connection between the DLPFC - amygdala circuitry. It might be that other brain regions, such as the rostral anterior cingulate cortex, play a crucial role in the effect of anxiety on the resolution of an emotional conflict (e.g., see work of Etkin, Egner, Peraza, Kandel, \& Hirsch, 2006). These latter researchers point to brain activation in the rostral anterior cingulate cortex in resolving emotional conflict, by decreasing the amygdala engagement to emotional distractors. In contrast, the right DLPFC was more involved in tracking the amount of distraction from emotional stimuli (Etkin et al., 2006). These research findings suggest that the DLPFC is only involved in down-regulating the amygdala when participants are 
explicitly asked to control their emotions. This is, however, not in line with Bishop et al. (2004a) who stated a key role of the DLPFC in down-regulating the amygdala during the presentation of threatening information, when no emotion regulation was asked for. Moreover, previous fMRI research in adolescents with anxiety disorders has shown an inverse correlation between anxiety symptoms and ventrolateral prefrontal cortex (VLPFC) activation during a dot probe task with threatening stimuli (e.g. Monk et al. 2006). Although prefrontal and anterior cingulate regions are highly inter-wired, future (rTMS) research should investigate the precise role of those brain regions implicated in attentional control to resolve emotional conflict from threatening information. This is important to fine grain prefrontal and/or anterior cingulate neural mechanisms in down-regulating amygdala activation that is responsible for anxiety and attentional bias (Bishop, Duncan, \& Lawrence, 2004b).

In conclusion, the present findings reveal that the ability of HF-rTMS of the right DLPFC to induce an attentional bias toward negative information in healthy individuals is influenced by the state anxiety level of that individual before the administration of rTMS. Future research should include participants with higher state and/or trait anxiety in order to investigate the specific effects in high and low anxiety to explore the neural and cognitive working mechanisms of rTMS as a treatment for pathological anxiety. 


\section{Acknowledgement}

MAV (FWO08/PDO/168) is a postdoctoral fellow of the Research Foundation Flanders (FWO). The authors are grateful for the help of Jerome Tullo with scientific interpretation and the drafting of this paper. The authors declare no conflicts of interest. 


\section{References}

Baeken, C., Leyman, L., De Raedt, R., Vanderhasselt, M.A., \& D’haenen, H. (2006). Lack of impact of repetitive high frequency transcranial magnetic stimulation on mood in healthy female subjects. Journal of Affective Disorders, 90, 63-66.

Baeken, C., Leyman, L., De Raedt, R., Vanderhasselt, M.A., \& D’haenen, H. (2008). Left and right high frequency repetitive transcranial magnetic stimulation of the dorsolateral prefrontal cortex does not affect mood in female volunteers. Clinical Neurophysiology, $119,568-575$.

Baeken, C., Vanderhasselt, M.A., \& De Raedt, R. (2010). Baseline 'state anxiety’ influences HPA-axis sensitivity to one sham-controlled HF-rTMS session applied to the right dorsolateral prefrontal cortex. Psychoneuroendocrinology. in press.

Bar-Haim, Y. (2010). Research Review: attention bias modification (ABM): a novel treatment for anxiety disorders. Journal of Child Psychology and Psychiatry, 51(8), 859-870.

Beck, A. T., Steer, R. A., \& Brown, G.K. (1996). Manual for the Beck Depression Inventory, second edition. San Antonio, TX: The Psychological Corporation. Dutch translated by van der Does, 2002.

Bishop, S.J., Duncan, J., \& Lawrence, A.D. (2004a). Prefrontal cortical function and anxiety: controlling attention to threat-related stimuli. Nature Neuroscience, 7(2), 184-188.

Bishop, S.J., Duncan, J., \& Lawrence, A.D. (2004b). State anxiety modulation of the amygdala response to unattended threat-related stimuli. Journal of Neuroscience, 24(46), 1036410368. 
Boggio, P. S., Rocha, M., Oliveira, M. O., Fecteau, S., Cohen, R. B., Campanha, C., \& Fregni, F. (2010). Noninvasive Brain Stimulation With High-Frequency and Low-Intensity Repetitive Transcranial Magnetic Stimulation Treatment for Posttraumatic Stress Disorder. Journal of Clinical Psychiatry, 71(8), 992-999.

Browning, M., Holmes, E. A., Murphy, S. E., Goodwin, G. M., \& Harmer, C. J. (2009). Learning to Look on the Bright Side: The Neural Consequences of an Induced Attentional Bias. Biological Psychiatry, 65(8), 276.

Cisler, J. M., \& Koster, E.H.W. (2010). Mechanisms of attentional biases towards threat in anxiety disorders: An integrative review. Clinical Psychology Review, 30(2), 203-216.

Cohen, J. (1988). Statistical power analysis for the behavioral sciences (Rev. ed.). Hillsdale, NJ: Erlbaum.

Davidson, R. J. (2002). Anxiety and affective style: Role of prefrontal cortex and amygdala. Biological Psychiatry, 51(1), 68-80.

De Raedt, R., Leyman, L., Baeken, C., Van Schuerbeek, P., Luypaert, R., Vanderhasselt, M.A., \& Dannlowski, U. (2010). Neurocognitive Effects of Hf-rTMS over the Dorsolateral Prefrontal Cortex on the Attentional Processing of Emotional Information in healthy women: An Event-Related fMRI Study. Biological Psychology, 85, 487-495.

De Raedt, R., \& Koster, E. H. W. (2010). Understanding vulnerability for depression from a cognitive neuroscience perspective: A reappraisal of attentional factors and a new conceptual framework. Cognitive Affective \& Behavioral Neuroscience, 10(1), 50-70. 
Dresler, T., Ehlis, A. C., Plichta, M. M., Richter, M. M., Jabs, B., Lesch, K. P., \& Fallgatter, A. J. (2009). Panic disorder and a possible treatment approach by means of high-frequency rTMS: A case report. World Journal of Biological Psychiatry, 10(4), 991-997.

Etkin, A., Egner, T., Peraza, D. M., Kandel, E. R., \& Hirsch, J. (2006). Resolving emotional conflict: A role for the rostral anterior cingulate cortex in modulating activity in the amygdala. Neuron, 51(6), 871-882. doi: 10.1016/j.neuron.2006.07.029

Fales, C. L., Barch, D. M., Rundle, M. M., Mintun, M. A., Snyder, A. Z., Cohen, J. D., Sheline, Y. I. (2008). Altered emotional interference processing in affective and cognitive-control brain circuitry in major depression. Biological Psychiatry, 63(4), 377-384.

Fox, E., Russo, R., \& Dutton, K. (2002). Attentional bias for threat: Evidence for delayed disengagement from emotional faces. Cognition \& Emotion, 16(3), 355-379.

Fox, E., Russo, R., \& Georgiou, G.A. (2005). Anxiety modulates the degree of attentive resources required to process emotional faces. Cognitive, Affective, \& Behavioural Neuroscience; 5(4), 396-404.

Fox, E., Russo, R., Bowles, R.J., \& Dutton, K. (2001). Do threatening stimuli draw or hold attention in subclinical anxiety? Journal of Experimental Psychology: General, 130, 681700.

Georgiou, G.A., Bleakley, C., Hayward, J., Russo, R., Dutton, K., Eltiti, S., \& Fox, E. (2005). Focusing on fear: Attentional disengagement from emotional faces. Visual Cognition, 12, $145-158$.

Goeleven, E., De Raedt, R., Leyman, L., \& Verschuere, B. (2008). The Karolinska directed emotional faces: a validation study. Cognition \& Emotion, 22(6), $1094-1118$. 
Hakamata, Y., Lissek, S., Bar-Haim, Y., Britton, J. C., Fox, N. A., Leibenluft, E., Ernst, M., Pine, D. S. (2010). Attention bias modification treatment: a meta-analysis toward the establishment of novel treatment for anxiety. Biological psychiatry, 68(11), 982-90.

Killgore, W.D., \& Yurgelun-Todd, D.A. (2001). Sex differences in amygdala activation during the perception of facial affect. Neuroreport. 12, 2543-2547.

Koster, E.H.W., De Raedt, R., Goeleven, E., Franck, E., \& Crombez, G. (2005). Moodcongruent attentional bias in dysphoria: Maintained attention to and impaired disengagement from negative information. Emotion, 5(4), 445-466.

Koster, E.H.W., Leyman, L., De Raedt, R., \& Crombez, G. (2006). Cueing of visual attention by emotional facial expressions: The influence of individual differences in anxiety and depression. Personality and Individual Differences, 41, 329-339.

Leyman, L., De Raedt, R., Vanderhasselt, M. A., \& Baeken, C. (2009). Influence of highfrequency repetitive transcranial magnetic stimulation over the dorsolateral prefrontal cortex on the inhibition of emotional information in healthy volunteers. Psychological Medicine, 39(6), 1019-1028.

Leyman, L., De Raedt, R., Schacht, R., \& Koster, E.H.W. (2007). Attentional biases for angry faces in unipolar depression. Psychological Medicine, 37(3), 393-402.

Leyman, L., De Raedt, R., Vanderhasselt, M. A., \& Baeken, C. (2011). Effects of repetitive transcranial magnetic stimulation of the dorsolateral prefrontal cortex on the attentional processing of emotional information in major depression: A pilot study. Psychiatry Research, 185, 102-107. 
Loo, C. K., Taylor, J. L., Gandevia, S.C., McDarmont, B.N., Mitchell, P.B., \& Sachdev, P.S. (2000). Transcranial magnetic stimulation (TMS) in controlled treatment studies: Are some "sham" forms active? Biological Psychiatry, 47(4), 325-331.

Lundqvist, D., Flykt, A. \& Öhman, A. (1998). The Karolinska Directed Emotional Faces $(K D E F)$. Stockholm: Department of Neurosciences, Karolinska Hospital.

MacLeod, C., Rutherford, E., Campbell, L., Ebsworthy, G., \& Holker, L. (2002). Selective attention and emotional vulnerability: Assessing the causal basis of their association through the experimental manipulation of attentional bias. Journal of Abnormal Psychology, 111(1), 107-123.

Monk, C. S., Nelson, E. E., McClure, E. B., Mogg, K., Bradley, B. P., Leibenluft, E., \& Pine, D. S. (2006). Ventrolateral prefrontal cortex activation and attentional bias in response to angry faces in adolescents with generalized anxiety disorder. American Journal of Psychiatry, 163(6), 1091-1097.

Mosimann, U.P., Rihs, T.A., Engeler, J., Fish, H., \& Schlaepfer, T.E. (2000). Mood effects of repetitive transcranial magnetic stimulation of left prefrontal cortex in healthy volunteers. Psychiatry Research, 94, 251-256.

Nitschke, J. B., \& Heller, W. (2005). Distinguishing neural substrates of heterogeneity among anxiety disorders. Neuroimaging, Pt B, 67, 1-+. doi: 10.1016/s0074-7742(05)67001-8.

Ochsner, K. N., Bunge, S. A., Gross, J. J., \& Gabrieli, J. D. E. (2002). Rethinking feelings: An fMRI study of the cognitive regulation of emotion. Journal of Cognitive Neuroscience, 14(8), 1215-1229. 
Pallanti, S., \& Bernardi, S. (2009). Neurobiology of repeated transcranial magnetic stimulation in the treatment of anxiety: a critical review. International Clinical Psychopharmacology, 24(4), 163-173.

Pascual-Leone, A., Tormos, J.M., Keenan, J., Tarazona, F., Canete, C., \& Catala, M.D. (1998).

Study and modulation of human cortical excitability with transcranial magnetic stimulation. Journal of Clinical Neurophysiology, 15(4), 333-343.

Peleman, K., Van Schuerbeek, P., Luypaert, R., Stadnik, T., De Raedt, R., De Mey, J., Bossuyt, A., \& Baeken, C. (2010). Using 3D-MRI to localize the dorsolateral prefrontal cortex in TMS research. World Journal of Biological Psychiatry, 11, 425-430.

Posner, M. I. (1980). Orientation of attention. Quarterly Journal of Experimental Psychology, $32,3-25$.

Rossi, S., Ferro, M., Cincotta, M., Ulivelli, M., Bartalini, S., Miniussi, C., Giovannelli, F., \& Passero, S. A real electro-magnetic placebo (REMP) device for sham transcranial magnetic stimulation (TMS). Clinical Neuropsychology, 118(3), 709-716.

Sheehan, D.V., Lecubrier, Y., Sheehan, K.H., Janvs, J., Weiller, E., Keskiner, A., Schinka, J., Knapp, E., Sheehan, M.F., \& Dunbar, G.C. (1997). The validity of the Mini International Neuropsychiatric Interviews (MINI) according to the SCID-P and its reliability. European Psychiatry, 12, 232-241. Dutch translation by Van Vliet, Leroy \& Van Megen, 2000.

Siegle, G. J., Steinhauer, S. R., Thase, M. E., Stenger, V. A., \& Carter, C. S. (2002). Can't Shake that feeling: Event-Related fMRI Assessment of Sustained Amygdala Activity in 
Response to Emotional Information in Depressed Individuals. Biological Psychiatry, 52(7), 772-772.

Spielberger, C.D. (1983). Manual for the State-Trait Anxiety Inventory. Paolo Alto, CA: Consulting Psychologists Press.

Taylor, J.G., Fragopanagos, N.F. (2005). The interaction of attention and emotion. Neural Networks, 18(4):353-369.

Vanderhasselt, M.A., De Raedt, R., Baeken, C., Leyman, L., \& D'Haenen, H. (2006). The influence of rTMS over the left dorsolateral prefrontal cortex on Stroop task performance. Experimental Brain Research, 169(2), 279-282.

Van der Ploeg, H.M. (1982). De Zelf-Beoordelings Vragenlijst (STAI-DY). De ontwikkeling en validatie van een Nederlandstalige vragenlijst voor het meten van angst. Tijdschrift voor psychiatrie, 24(9), 576-588.

Van Strien A. (2001). Handvoorkeur en taaldominantie. Neuropraxis, 2, 10-15.

Wager, T.D., Phan, K.L., Liberzon, I., Taylor S.F. (2003). Valence, gender, and lateralization of functional brain anatomy in emotion: a meta-analysis of findings from neuroimaging. Neuroimage, 19, 513-531.

Wald, F.D.M., \& Mellenbergh, G.J. (1990). De verkorte versie van de Nederlandse vertaling van de Profile of Moods State (POMS). [The short Dutch version of the Profile of Moods State (POMS) questionnaire]. Nederlands Tijdschrift voor de Psychologie, 45, 86-90. 
Table 1

Mean scores (and standard deviations) on all five POMS subscales pre (T0), immediately after (T1) and half an hour after (T2) rTMS and SHAM stimulation

\begin{tabular}{|c|c|c|c|c|c|c|}
\hline \multirow[b]{2}{*}{$\begin{array}{c}\text { POMS } \\
\text { Subscale }\end{array}$} & \multicolumn{3}{|c|}{ rTMS } & \multicolumn{3}{|c|}{ SHAM } \\
\hline & $\mathrm{T}_{0}$ & $\mathrm{~T}_{1}$ & $\mathrm{~T}_{2}$ & $\mathrm{~T}_{0}$ & $\mathrm{~T}_{1}$ & $\mathrm{~T}_{2}$ \\
\hline Depression & $0.48(1.34)$ & $0.13(0.46)$ & $0.22(0.60)$ & $0.61(1.27)$ & $0.26(0.62)$ & $0.30(0.93)$ \\
\hline Anger & $0.74(1.48)$ & $0.57(2.11)$ & $0.30(0.76)$ & $0.65(1.5)$ & $0.13(0.46)$ & $0.09(0.42)$ \\
\hline Tension & $1.26(1.48)$ & $0.61(1.34)$ & $0.61(0.94)$ & $0.87(.87)$ & $0.48(1.2)$ & $0.52(1.16)$ \\
\hline Fatigue & $4.04(3.97)$ & $2.78(2.92)$ & $3.52(3.27)$ & $4.87(5.56)$ & $3(2.81)$ & $3.43(4.01)$ \\
\hline Vigor & $10.3(4.79)$ & $9.22(4.55)$ & $8.43(4.58)$ & $8.04(4.65)$ & $8.39(4.29)$ & $7.96(5.11)$ \\
\hline
\end{tabular}

Table 2

Mean reaction time data (and standard deviations) (in ms) for valid and invalid ECT trials, pre and post rTMS and SHAM stimulation

\begin{tabular}{|c|c|c|c|c|c|}
\hline \multirow[b]{2}{*}{ Valence } & \multirow[b]{2}{*}{ Trial Type } & \multicolumn{2}{|c|}{ rTMS } & \multicolumn{2}{|c|}{ SHAM } \\
\hline & & pre & post & pre & post \\
\hline \multirow[t]{2}{*}{ Angry } & Valid & $376(63)$ & $361(51)$ & 375 (57) & $356(55)$ \\
\hline & Invalid & $401(64)$ & $411(57)$ & $400(58)$ & $387(66)$ \\
\hline \multirow[t]{2}{*}{ Neutral } & Valid & $375(61)$ & $356(50)$ & $374(53)$ & $351(57)$ \\
\hline & Invalid & $405(65)$ & $386(60)$ & 408 (69) & $385(67)$ \\
\hline
\end{tabular}




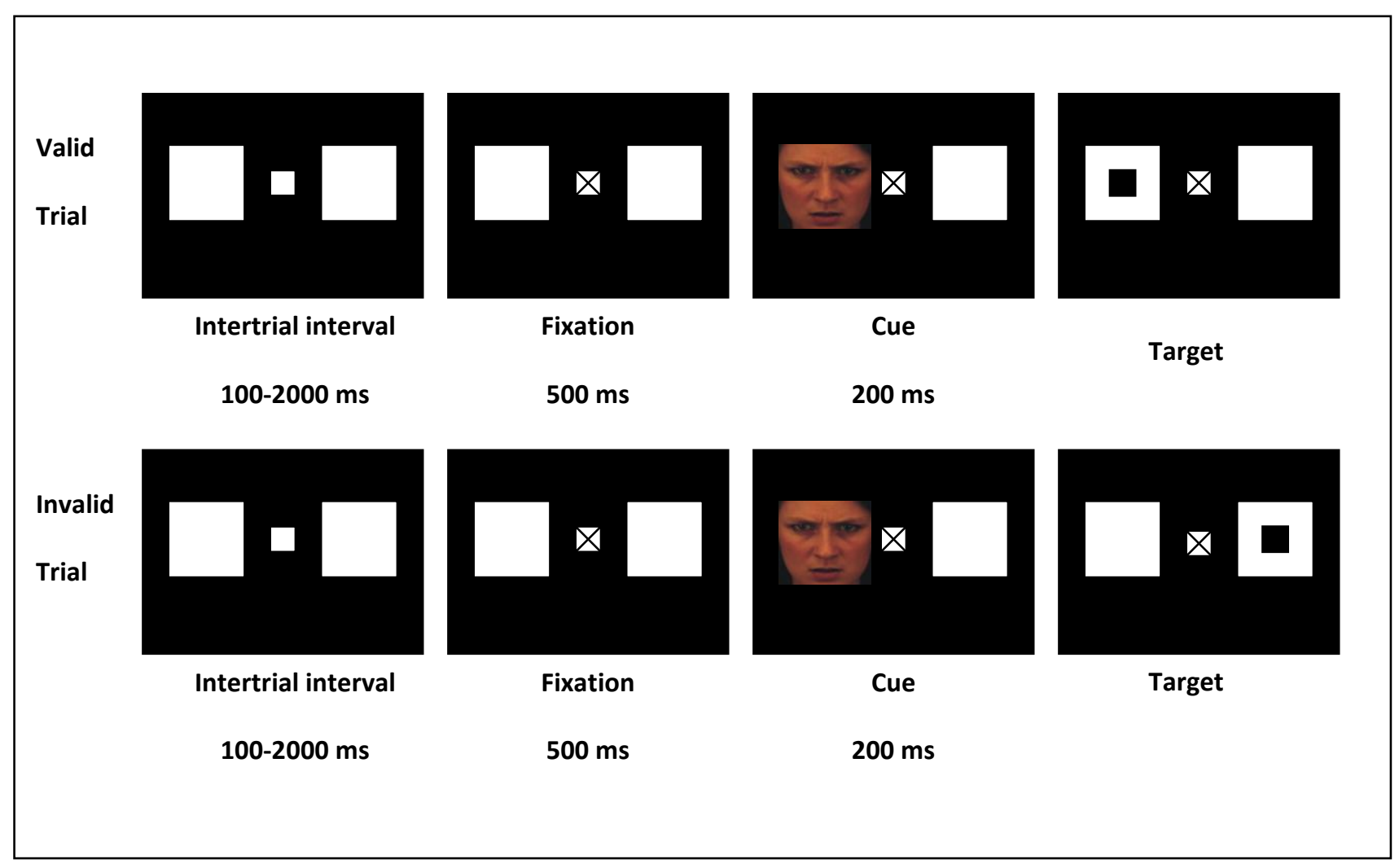

Figure A1. The Exogenous cueing task: Stimulus presentation on valid and invalid trials. 


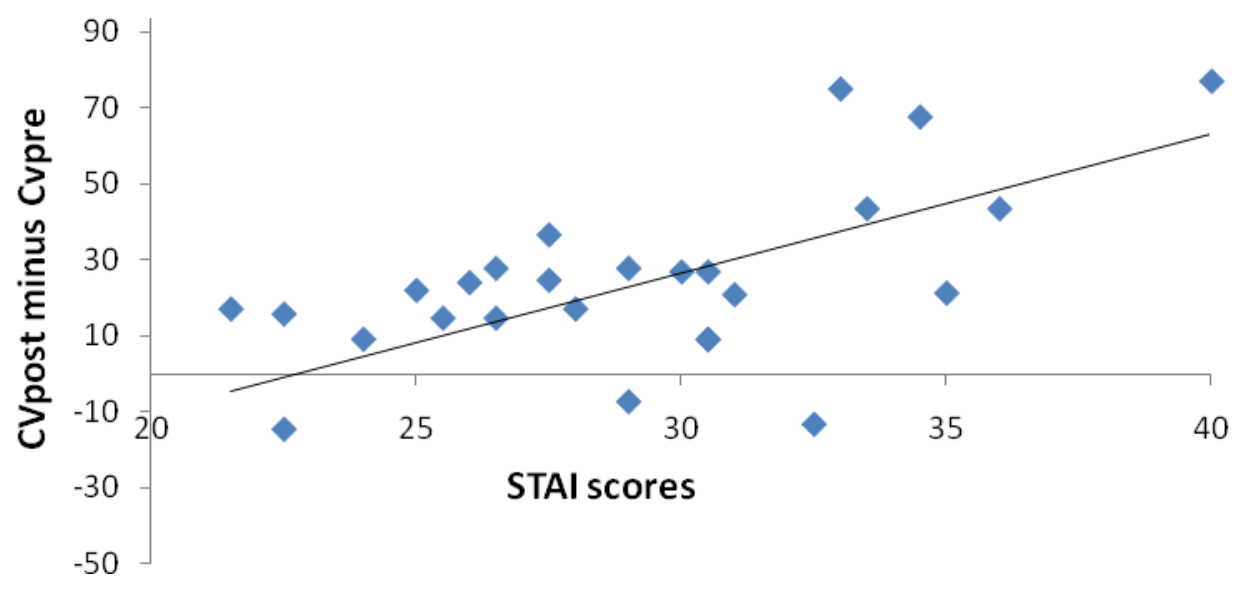

Figure A2. Scatter plot demonstrating a correlation between STAI scores with the difference in attentional bias scores pre-post rTMS, $r(28)=.53 ; p<.01$. 